\title{
DEGREES OF EXCEPTIONAL CHARACTERS OF CERTAIN FINITE GROUPS
}

BY

HARVEY I. BLAU

\begin{abstract}
Let $G$ be a finite group whose order is divisible by a prime $p$ to the first power only. Restrictions beyond the known congruences modulo $p$ are shown to hold for the degrees of the exceptional characters of $G$, under the assumptions that either all $p^{\prime}$-elements centralizing a Sylow $p$-subgroup are in fact central in $G$ and there are at least three conjugacy classes of elements of order $p$, or that the characters in question lie in the principal $p$-block. Results of Feit and the author are thereby generalized.
\end{abstract}

1. Introduction. This paper proves two theorems on the possible degrees of irreducible characters of some finite groups whose orders are divisible by a prime to the first power only. We first set some notation and hypotheses, and recall a few basic results.

$G$ denotes a finite group, $p$ an odd prime, $P$ a Sylow $p$-subgroup of $G . N$ and $C$ are the normalizer, resp. centralizer, of $P$ in $G, e=|N: C|$ and $t=(p-1) / e . Z$ is the center of $G$. We follow the terminology of [6], [1] and say that $G$ is of type $L_{2}(p)$ if each of its composition factors is either a $p$-group, a $p^{\prime}$-group, or isomorphic to $P S L_{2}(p)$.

Assume that $|P|=p$, so that $t$ is the number of conjugacy classes of elements of order $p$. Suppose that $C=P \times Z$. Then [5] implies the following: for any $p$-block $B$ of positive defect there is a sign $\delta_{B}= \pm 1$ such that the degree of all the exceptional characters in $B$ is congruent to $\delta_{B} e(\bmod p)$. The degrees of the nonexceptional characters in $B$ are congruent to \pm 1 ( mod $p$ ). Further, there is a one-to-one correspondence between $p$-blocks of positive defect and irreducible (linear) characters $\eta$ of $Z$ : an irreducible character $\chi$ is in $B_{\eta}$ if and only if $p \nmid \chi(1)$ and $\chi_{Z}=\chi(1) \eta$. It follows that if $\chi$ and $\xi$ are in $p$-blocks of positive defect, then all the irreducible constituents of $\chi \xi$ that are not of defect zero lie in a unique $p$-block, which we denote by $B(\chi \xi) . B_{1}$ will mean the principal $p$-block.

Theorem 1. Assume that $G=G^{\prime}$ is not of type $L_{2}(p), C=P \times Z,|P|=p$ and $t \geqslant 3$. Let $\chi, \xi$ be exceptional characters of $G$ in $p$-blocks of positive defect,

Received by the editors November 23, 1976.

AMS (MOS) subject classifications (1970). Primary 20C15; Secondary 20C20, $20 \mathrm{D} 05$.

Key words and phrases. Ordinary character, exceptional character, modular representation, cyclic Sylow subgroup, symmetric and skew decomposition, principal block.

(C) 1979 American Mathematical Society 0002-9947/79/0000-0152/\$04.00 
with either $\chi(1)=n p+e$ and $\xi(1)=m p+e$ or $\chi(1)=n p-e$ and $\xi(1)=m p$ - $e$ for some positive integers $n, m$. Let $B=B(\chi \xi)$. If $\delta_{B}=-1$, then there is a function $f(n, m)$, independent of $p$, such that $e \leqslant f(n, m)$. If $\delta_{B}=+1$, then there is a function $f(n, m, t)$ (depending on $n, m$, and $t$ but not on $p)$ such that $e \leqslant f(n, m, t)$.

Note that $[8,1.17 .13]$ and $[6$, Theorem 1] imply that when $\chi(1) \equiv \xi(1) \equiv e$ $(\bmod p)$, the assumption that $G$ is not of type $L_{2}(p)$ forces $m$ and $n$ to be positive.

If $\chi(1)=p-e=\xi(1)$, then if $\delta_{B}=-1$ we must have $e=2$ (and if $\chi$ or $\xi$ is faithful, the assumption $C=P \times Z$ is redundant) [7, (4.1), (2.1)], [3, Theorem 1 and Remark (iii)]. If $\delta_{B}=+1$ then $e \leqslant t-3$ [2], [3, Theorem 1 and Remark (iv)]. Theorem 1 may be considered a generalization of these results, but the upper bounds obtained for $e$, namely

$$
f(n, m)=4 n+4 m+16 n m, f(n, m, t)=n+m+n m(t+1)
$$

seem far from sharp. For instance, they do not quite reach the abovementioned bounds when $m=n=1$. Furthermore, there are no examples known where the hypotheses of Theorem 1 are satisfied with $\chi(1)=p-e=$ $\xi(1), \delta_{B}=+1$, and $e>2$. However, we obtain a nearly best possible result in

THEOREM 2. Assume that $G=G^{\prime},|P|=p$, and that $G$ has an irreducible character $\chi \in B_{1}$ with $(3 p+1) / 2<\chi(1)<2 p-1$. Then $\chi(1)$ equals $2 p-2$ or $2 p-4$.

The case $\chi(1)=2 p-2$ occurs. For example, $P S L_{2}(25)$ has a faithful character of degree 24 in the principal 13-block. However, I do not know if there is an instance of the case $\chi(1)=2 p-4$ under the hypotheses of Theorem 2. The theorem is almost a "translation $p$ units to the right" of a special case of [7, Theorem 1], namely, that if $(p+1) / 2<\chi(1)<p-1$, and the other hypotheses of Theorem 2 hold, then $\chi(1)=p-2$. Theorem 2 contains no assumption on $C$, but note (3.1) of the proof.

Analogs of our theorems may hold without the assumptions $C=P \times Z$ or $\chi \in B_{1}$. However, their proofs would involve extensions of the available machinery which $I$ have not yet completed. Theorems 1 and 2 are proved below with the techniques of [7], [1], and [3].

\section{Proof of Theorem 1.}

Notation. $R$ is the ring of integers in a $p$-adic number field $F$ such that $F$ and $R / J(R)$ are splitting fields for all subgroups of $G . R^{\prime}$ is a finite extension of $R$ with ramification index at least $2\left[8\right.$, p. 103]. $K=R^{\prime} / J\left(R^{\prime}\right) . Y^{*}$ is the dual of a $K G$-module $Y, \zeta^{*}$ the complex conjugate of a complex character $\zeta$. Since $C=P \times Z$ implies $N / P$ is abelian, the indecomposable $K N$-modules are described in $[1, \S 2]$. We freely use the notation and terminology of [1]. In 
particular, if $N=P H$ with $H$ a $p^{\prime}$-group, then char $H$ is the set of all linear characters: $H \rightarrow K$. The particular character $\alpha \in \operatorname{char} H$ is defined by

$$
h^{-1} y h=y^{\alpha(h)}
$$

for all $h \in H$, where $\langle y\rangle=P$. A typical indecomposable $K N$-module is $V_{m}(\lambda), 1 \leqslant m \leqslant p, \lambda \in$ char $H$. If $X$ is a $K G$-module with $X_{N}=\bigoplus \Sigma_{i} V_{d_{i}}\left(\lambda_{i}\right)$, the $H$-values of $X$ are all the $\lambda_{i} \alpha^{-j}, 0 \leqslant j \leqslant d_{i}-1$ [1, Proposition 2.11]. The main values are the $\lambda_{i}$. Such a $\lambda_{i}$ is a projective main value (pmv) of $X$ if $d_{i}=p$, a nonprojective main value (npmv) if not (a given character could be both). If $X$ is nonprojective and indecomposable, $X \leftrightarrow V_{m}(\lambda)$ means that $V_{m}(\lambda)$ is the Green correspondent of $X$ (so $V_{m}(\lambda) \mid X_{N}$ and $\lambda$ is the unique npmv of $X$ ) [8, III.5.6].

In addition to all the hypotheses of Theorem 1, we may assume $e>2$. Let $L^{\prime}, M^{\prime}$ be $R^{\prime}$-free, $R^{\prime} G$-modules affording $\chi, \xi$ resp. such that $L=$ $L^{\prime} / J\left(R^{\prime}\right) L^{\prime}$ and $M=M^{\prime} / J\left(R^{\prime}\right) M^{\prime}$ are indecomposable [8, I.17.13]. Let $\nu= \pm 1$ such that $\chi(1) \equiv \xi(1) \equiv \nu e(\bmod p)$. The Green correspondence implies that when $\nu=1$,

$$
L_{N}=V_{e}(\lambda) \oplus \sum_{\sigma \in \mathcal{S}} V_{p}(\sigma), \quad M_{N}=V_{e}(\mu) \oplus \sum_{\tau \in \mathscr{T}} V_{p}(\tau)
$$

for some $\lambda, \mu \in$ char $H$, where $\mathcal{S}, \mathcal{T}$ are subsets of char $H$, counting possible multiplicities, with $|\delta|=n,|\mathcal{T}|=m$. If $\nu=-1$,

$$
L_{N}=V_{p-e}(\lambda) \oplus \sum_{\sigma \in \delta} V_{p}(\sigma), \quad M_{N}=V_{p-e}(\mu) \oplus \sum_{\tau \in \mathcal{T}} V_{p}(\tau)
$$

where $|\delta|=n-1,|\mathcal{T}|=m-1$. Then [1, Lemmas 2.4, 2.5] (and the fact $\alpha^{e}=1$ ) imply that when $\nu=1$,

$$
\begin{aligned}
\left(L \otimes_{K} M\right)_{N}= & \oplus \sum_{i=0}^{e-1} V_{2 i+1}\left(\lambda \mu \alpha^{1+i}\right) \oplus \sum_{\sigma \in \mathcal{S}} \sum_{i=0}^{e-1} V_{p}\left(\mu \sigma \alpha^{-i}\right) \\
& \oplus \sum_{\tau \in \mathcal{T}} \sum_{i=0}^{e-1} V_{p}\left(\lambda \tau \alpha^{-i}\right) \oplus \sum_{\mathcal{S}, \mathscr{T}} \sum_{i=0}^{p-1} V_{p}\left(\sigma \tau \alpha^{-i}\right) .
\end{aligned}
$$

When $\nu=-1,[1$, Lemmas $2.5,2.6]$ yields

$$
\begin{aligned}
\left(L \otimes_{K} M\right)_{N}= & \oplus \sum_{i=0}^{e-1} V_{2 i+1}\left(\lambda \mu \alpha^{i}\right) \oplus \sum_{i=0}^{p-2 e-1} V_{p}\left(\lambda \mu \alpha^{-i}\right) \\
& \oplus \sum_{\sigma \in \delta} \sum_{i=0}^{p-e-1} V_{p}\left(\mu \sigma \alpha^{-i}\right) \\
& \oplus \sum_{\tau \in \mathcal{T}} \sum_{i=0}^{p-e-1} V_{p}\left(\lambda \tau \alpha^{-i}\right) \oplus \sum_{\delta, \mathcal{T}} \sum_{i=0}^{p-1} V_{p}\left(\sigma \tau \alpha^{-i}\right) .
\end{aligned}
$$

Let $L_{i} \leftrightarrow V_{2 i+1}\left(\lambda \mu \alpha^{1+i}\right)$ when $\nu=1, L_{i} \leftrightarrow V_{2 i+1}\left(\lambda \mu \alpha^{i}\right)$ when $\nu=-1$. The 
Green correspondence implies

$$
L \otimes M=\oplus \sum_{i=0}^{e-1} L_{i} \oplus Q
$$

where $Q$ is projective.

Now since $|\langle\alpha\rangle|=e$, any $\gamma \in$ char $H$ can occur at most $[u / e]+1$ times as a pmv of $\bigoplus \Sigma_{i=0}^{u} V_{p}\left(\rho \alpha^{-i}\right)$ (where $\rho$ is fixed in char $H$ and square brackets denote the greatest integer symbol). Then (2.1) and (2.2) imply

(2.3) Any $\gamma \in$ char $H$ can occur as a pmv of $L \otimes M$ at most $n+m+n m(t$ $+1)$ times if $\nu=1$, and at most $t-1+(n-1) t+(m-1) t+(n-1)(m-$ $1)(t+1)=n m(t+1)-n-m$ times if $\nu=-1$.

For each $i$ with $0 \leqslant i \leqslant e / 2-1$ ( $e$ even), or $0 \leqslant i \leqslant(e-3) / 2$ ( $e$ odd), there is an $R^{\prime}$-free, $R^{\prime} G$-module $X_{i}$ such that

$$
X_{i} / J\left(R^{\prime}\right) X_{i} \approx L_{i} \oplus L_{e-1-i} \quad[3, \text { Lemma 2.1] }
$$

Also, there is an $R^{\prime}$-free, $R^{\prime} G$-module $Y$ with $Y / J\left(R^{\prime}\right) Y \approx L_{e / 2}(e$ even) or $Y / J\left(R^{\prime}\right) Y \approx L_{(e-1) / 2}(e$ odd). Now the exceptional characters in $B$ occur in the character afforded by each $X_{i}$ with total multiplicity at least 2 if $\delta_{B}=1$, and at least $t-2$ if $\delta_{B}=-1$. They occur in the character afforded by $Y$ with total multiplicity at least 1 if $\delta_{B}=1$, and at least $t-1$ if $\delta_{B}=-1$ [3, Lemma 2.2].

Let $U$ be an irreducible $K G$-module which is a modular constituent of an exceptional character in $B$. Then $U$ is a constituent of $L_{i} \oplus L_{e-1-i}, L_{e / 2}$ or $L_{(e-1) / 2}$ with multiplicity at least each of the above, resp. So if $\delta_{B}=+1$, then $U$ occurs in $\bigoplus \sum_{i=0}^{e-1} L_{i}$ at least $2(e / 2)=e$ times if $e$ is even, and at least $2(e-1) / 2+1=e$ times if $e$ is odd. If $\delta_{B}=-1$, then $U$ is a constituent of $\bigoplus \sum_{i=0}^{e-1} L_{i}$ at least $(t-2)(e / 2-1)+(t-1)=e(t-2) / 2+1$ times if $e$ is even, and at least $(t-2)(e-1) / 2+(t-1)=e(t-2) / 2+t / 2$ times if $e$ is odd.

Suppose $\delta_{B}=1$. By the argument of $[3, \mathrm{p}$. 552] (where it suffices that $e \leqslant(p-1) / 3)$, there exists a module $U$, as above, with a pmv $\pi$. Thus $\pi$ occurs at least $e$ times as a pmv of $L \otimes M$. Now (2.3) implies $e \leqslant n+m+$ $n m(t+1)$. Theorem 1 is proved in this case, with

$$
f(n, m, t)=n+m+n m(t+1) .
$$

Suppose $\delta_{B}=-1$. The argument of [3, p. 553] shows that there exists a modular constituent $U$ (with npmv $\beta$, say) of the exceptional characters in $B$ such that if there is an irreducible $K G$-module $W$ which absorbs $\beta$ (see [3, p. 546]), then $W$ has a pmv $\gamma$. If $W$ does not exist, or if $\gamma=\beta$, then [3, Lemma 2.7] implies that $\beta$ is a pmv of $L \otimes M$ at least $e(t-2) / 2$ times. Then by (2.3), 


$$
e(t-2) / 2 \leqslant n+m+n m(t+1)
$$

whence

$$
e \leqslant(n+m+n m(t+1))(2 /(t-2)) \leqslant 2 n+2 m+8 n m
$$

since $t \geqslant 3$. If $W$ exists with $\gamma \neq \beta$, then [3, Lemma 2.7] yields that $\gamma, \beta$ occur together as pmv's of $L \otimes M$ at least $e(t-2) / 2$ times. Then (2.3) yields

$$
e(t-2) / 2 \leqslant 2(n+m+n m(t+1)),
$$

so that

$$
e \leqslant(n+m+n m(t+1))(4 /(t-2)) \leqslant 4 n+4 m+16 n m .
$$

We take $f(n, m)=16 n m+4 n+4 m$ to complete the proof of Theorem 1 .

REMARK. The bounds may be sharpened by handling the small values of $t$, or the cases $\nu= \pm 1$, separately. They may also be improved if we know that $\lambda$, $\mu$, and the linear characters in $\delta$ and $\mathcal{T}$ are not all identical (they usually are not).

3. Proof of Theorem 2. Assume that $G$ and $\chi$ satisfy the hypotheses of Theorem 2. By [5, Theorem 11], the degrees of the characters in $B_{1}$ are congruent to \pm 1 or $\pm e(\bmod p)$. Then $(3 p+1) / 2<\chi(1)<2 p-1$ implies $\chi(1)=2 p-e$ where $1<e<(p-1) / 2$, so that $\chi$ is an exceptional character in $B_{1}$.

(3.1) It suffices to assume $\chi$ is faithful, $C=P$ and char $H=\langle\alpha\rangle$.

Proof. Let $J$ be the kernel of $\chi$. If $J \supseteq P$, then $\chi$, one of $t \geqslant 3 p$-conjugate characters, is invariant under $p$-conjugation, a contradiction. So $J$ is a $p^{\prime}$-group and $B_{1}(G / J)=B_{1}(G)$ [8, IV.4.12, V.4.3]. Therefore, $\chi \in B_{1}(G / J)$, and the hypotheses of Theorem 2 are satisfied for group $G / J$ and character $\chi$. So we may assume $J=\langle 1\rangle$. Then $C=P[8, I V .4 .21]$. So $N / P$ is cyclic and char $H=\langle\alpha\rangle$.

(3.2) $G$ is simple.

Proof. $O_{p^{\prime}}(G)$ is the kernel of $B_{1}$ [8, IV.4.12], so $\chi$ faithful implies $O_{p^{\prime}}(G)=\langle 1\rangle$. Thus if $J$ is a proper normal subgroup of $G$, then $P \subseteq J$. The Frattini argument implies $G=J N$. Then $G / J \approx N / N \cap J$ is a homomorphic image of $N / P$, and hence is cyclic. Then $G^{\prime} \subseteq J$ yields $J=G$.

(3.3) The assumptions of Theorem 1 hold with $\chi=\xi, L=M, \chi(1)=2 p-e$, $B=B_{1}$, and $\delta_{B}=-1$.

Proof. Since $e<(p-1) / 2, G \approx P S L_{2}(p)$. Then (3.2) says $G$ is not of type $L_{2}(p)$. The rest is clear.

(3.4) If $L$ is reducible it has exactly two constituents, say $U$ and $W$, where 
$\operatorname{dim} U=p-s, \operatorname{dim} W=p-e+s$, some $s<e$. Also, $U, W$ are both selfdual, and the node for $\chi$ (and the other exceptionals) in the Brauer graph of $B_{1}$ appears as

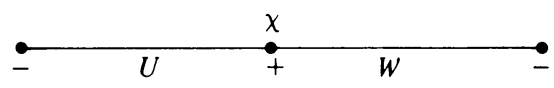

Furthermore, if $U_{N}=V_{p-s}(\beta)$ then $W_{N}=V_{p-e+s}\left(\beta \alpha^{s}\right)$.

Proof. $\chi(1) \equiv-e(\bmod p)$ implies that each modular constituent $U$ of $L$ has

$$
\operatorname{sep} U=-\operatorname{rem} U
$$

(see $[1, \S 4]$ and [9]). Thus $\operatorname{dim} U \geqslant p-e$. So $\chi(1)=2 p-e$ forces $L$ to have at most two (necessarily distinct) irreducible constituents, say $U$ and $W$. Suppose there are two. Then sep $U+\operatorname{sep} W=e, \operatorname{so} \operatorname{dim} U=p-s, \operatorname{dim} W$ $=p-e+s$, some $1 \leqslant s \leqslant e-1 . \chi$ lies on the real stem of $B_{1}$, and symmetry about the stem means that $U$ and $W$ are self-dual. Let $U_{N}=$ $V_{p-s}(\beta), W_{N}=V_{p-e+s}(\tau)$. We may assume there is an exact sequence $0 \rightarrow U \rightarrow L \rightarrow W \rightarrow 0$. Then [1, Proposition 4.5] implies $\tau=\beta \alpha^{p-e+s-1}=$ $\beta \alpha^{s}$.

(3.5) If $e$ is even then $L$ is irreducible and $L_{N}=V_{p-e}(-1) \oplus V_{p}(1)$, where $-1=\alpha^{e / 2}$.

Proof. Suppose $L$ is reducible. Let $U, W$ be as in (3.4) and $d=p-s$. $U \approx U^{*}$ implies $\beta^{2}=\alpha^{d-1}$ [1, Lemma 2.3]. Now $\beta \in\langle\alpha\rangle$, so $e$ even implies $d$ is odd. $G=G^{\prime}$ means that every representation of $G$ is unimodular. Then [1, Lemma 2.3] yields that $\beta^{d}=\alpha^{d(d-1) / 2}$. Since $d$ is odd, $\beta$ may be found from $\beta^{2}$ and $\beta^{d}$ : namely, $\beta=\alpha^{(d-1) / 2}$. A similar argument for $W$ implies

$$
\beta \alpha^{s}=\alpha^{(p-e+s-1) / 2}=\alpha^{(d-1) / 2} \alpha^{s} \alpha^{e / 2} .
$$

Hence, $\beta=\alpha^{(d-1) / 2} \alpha^{e / 2}$ so that $\alpha^{e / 2}=1$, a contradiction.

So $L$ is irreducible and must lie on the real stem. Let $L_{N}=V_{p-e}(\lambda) \oplus$ $V_{p}(\sigma)$. Then $L \approx L^{*}$ implies $\lambda^{2}=\alpha^{p-e-1}=1$. If $\lambda=1$ then $L \approx 1_{0}$ (the trivial one-dimensional $K G$-module) [1, Corollary 4.7], a contradiction. So $\lambda=-1$. Again, $G=G^{\prime}$ and [1, Lemma 2.3] imply

$$
1=\lambda^{p-e} \alpha^{-(p-e)(p-e-1) / 2} \sigma^{p} \alpha^{-p(p-1) / 2}=\sigma^{p}=\sigma .
$$

(3.6) Assume e is even. Let $S \oplus T$ denote the decomposition of $L \otimes L$ into symmetric and skew parts, respectively. Define $\gamma \in\langle\alpha\rangle$ to be even if $\gamma \in\left\langle\alpha^{2}\right\rangle$, odd if not. Then 


$$
\begin{gathered}
S_{N}=\bigoplus_{0<i<e-1 ; i=0(\bmod 2)} V_{2 i+1}\left(\alpha^{i}\right) \oplus(3 t-3) \sum_{\gamma \text { even; } \gamma \neq \pm 1} V_{p}(\gamma) \\
T_{N}=\bigoplus_{0<i<e-1 ; i \equiv 1(\bmod 2)} V_{2 i+1}\left(\alpha^{i}\right) \oplus(t-1) \sum_{\gamma \text { odd } ; \gamma \neq-1} V_{p}(\gamma) \oplus(3 t-1) V_{p}(1) \oplus x V_{p}(-1), \\
\oplus(3 t-3) \sum_{\gamma \text { odd } ; \gamma \neq-1} V_{p}(\gamma)
\end{gathered}
$$

where $x=3 t-2$ if $\alpha^{e / 2}$ is even, and $x=t$ if $\alpha^{e / 2}$ is odd.

Proof. $S$ and $T$ are $K G$-submodules of $L \otimes L$, and $S_{N}, T_{N}$ are the symmetric (resp. skew) summands of $(L \otimes L)_{N} . S$ has $K$-dimension $(2 p-$ $e)(2 p-e+1) / 2$ and

$$
\operatorname{dim} T=(2 p-e)(2 p-e-1) / 2
$$

(see $[1, \S 3])$. The $H$-values of $L_{N}$ are $\left\{\alpha^{(e / 2)-i} \mid 0 \leqslant i \leqslant p-e-1\right\} \cup\left\{\alpha^{-i} \mid 0\right.$ $\leqslant i \leqslant p-1\}$ by (3.5) and [1, Proposition 2.11]. Squaring each element of this set yields every even character $4 t-2$ times, plus the trivial character twice more. Then [1, (3.1)] implies

(3.7) The $H$-values of $S_{N}$ consist of the $H$-values of $T_{N}$, together with each nontrivial even character of $\langle\alpha\rangle$, with additional multiplicity $4 t-2$, and the trivial character, with additional multiplicity $4 t$.

Now by (2.2),

$$
\begin{aligned}
(L \otimes L)_{N}= & \oplus \sum_{i=0}^{e-1} V_{2 i+1}\left(\alpha^{i}\right) \oplus \sum_{i=0}^{p-2 e-1} V_{p}\left(\alpha^{-i}\right) \\
& \oplus 2 \sum_{i=0}^{p-e-1} V_{p}\left(\alpha^{(e / 2)-i}\right) \oplus \sum_{i=0}^{p-1} V_{p}\left(\alpha^{-i}\right) \\
= & \oplus \sum_{i=0}^{e-1} V_{2 i+1}\left(\alpha^{i}\right) \oplus(4 t-4) \sum_{\gamma \neq \pm 1} V_{p}(\gamma) \\
& \oplus(4 t-2) V_{p}(1) \oplus(4 t-2) V_{p}(-1) .
\end{aligned}
$$

The Krull-Schmidt theorem implies the summands in (3.8) are distributed between $S_{N}$ and $T_{N}$. Since $V_{p-e}(-1) \mid L_{N},[1,(3.1)]$ implies the symmetric (resp. skew) part of $V_{p-e}(-1) \otimes V_{p-e}(-1)$ is a summand of $S_{N}$ (resp. $T_{N}$ ). So by [1, Lemma 3.3],

$$
\bigoplus \sum_{0<i<e-1, i \equiv 0(\bmod 2)} V_{2 i+1}\left(\alpha^{i}\right)\left|S_{N}, \quad \bigoplus \sum_{0<i<e-1 ; i \equiv 1(\bmod 2)} V_{2 i+1}\left(\alpha^{i}\right)\right| T_{N} .
$$


Then a dimension argument implies $S_{N}$ must have exactly two more projective indecomposable summands than $T_{N}$ does.

Now (3.9) shows that from the nonprojective summands alone, each odd $\gamma$ occurs as an $H$-value of $T_{N}$ twice more than in $S_{N}$, and the even characters are balanced as $H$-values. Neglecting pmv's, the projective summands must contribute $2 t$ more of each character as an $H$-value of $S_{N}$ than as an $H$-value of $T_{N}$. So without the pmv's, $S_{N}$ has $2 t$ more of each even $\gamma$, and $2 t-2$ of each odd $\gamma$, occurring as $H$-values than $T_{N}$ does. Now (3.7) implies for each even $\gamma \neq 1, V_{p}(\gamma)$ must occur in $S_{N} 2 t-2$ more times than in $T_{N}$. Also, for each odd $\gamma, V_{p}(\gamma)$ is a summand of $T_{N} 2 t-2$ more times than in $S_{N}$. Finally, $V_{p}(1)$ occurs in $S_{N} 2 t$ more times than in $T_{N}$. The result follows from (3.8).

(3.10) If $e$ is even then e equals 2 or 4.

Proof. Suppose $e \geqslant 6$. Let $L_{i} \leftrightarrow V_{2 i+1}\left(\alpha^{i}\right), 0 \leqslant i \leqslant e-1$. Then by the proof of Theorem 1 (with $L=M$ ), $L$, the unique modular constituent of the exceptional characters in $B_{1}$, is a constituent of $L_{0} \oplus L_{e-1}$ with multiplicity at least $t-2$. Since $L_{0}=1_{0}$ (by the Green correspondence), $L$ is a constituent of $L_{e-1}$ at least $t-2$ times.

Because $e<2 e-5<p-e,[1$, Lemma 2.6] implies the npmv's of $V_{p-e}(-1) \otimes V_{2 e-5}\left(\alpha^{e-3}\right)$ are

$$
\alpha^{e / 2} \alpha^{e-3} \alpha^{-(2 e-5)+1+i}=\alpha^{(e / 2)+3+i,} \quad 0 \leqslant i \leqslant e-1 .
$$

Hence, 1 is a npmv of $L \otimes L_{e-3} \approx L^{*} \otimes L_{e-3} \approx \operatorname{Hom}_{K}\left(L, L_{e-3}\right)$. By [1, Theorem 4.1], there is a nonzero $K G$-homomorphism from irreducible $L$ into indecomposable $L_{e-3} \not L$. Since $L$ and $L_{e-3}$ are both self-dual, [1, Proposition 4.11] shows that $L$ is a constituent of $L_{e-3}$ with multiplicity at least 2.

Now $L_{e-1} \oplus L_{e-3} \mid T$ by (3.6) and the Green correspondence. Hence, $L$ is a constituent of $T$ with multiplicity at least $t$, so that 1 (the pmv of $L$ ), is a pmv of $T$ with multiplicity at least $t$. But (3.6) also implies 1 has multiplicity exactly $t-1$ as a pmv of $T$, a contradiction.

(3.11) If $e$ is odd then $e=3,9$, or $15, L$ is reducible, and for $U_{N}=V_{p-s}(\beta)$ as in (3.4), we may assume $s=2 e / 3$ and $\beta=\alpha^{s}$.

Proof. Suppose $L$ is irreducible. $L \approx L^{*}$ implies $V_{p-e}(\lambda) \approx V_{p-e}(\lambda)^{*}$, hence $\lambda^{2}=\alpha^{p-e-1}=1$ [1, Lemma 2.3]. Then $e$ odd yields $\lambda=1$. Thus $L \approx 1_{0}[1$, Corollary 4.7], a contradiction.

Let $U, W$ be as in (3.4). Since $\chi(1)$ is odd, we may assume $s$ is even (if not, $e-s$ is). Then $U \approx U^{*}$ implies $\beta^{2}=\alpha^{p-s-1}$, whence $\beta=\alpha^{-s / 2}$. Now $|H|=e$. Since $H$ is a $p^{\prime}$-group, we may identify $\alpha(H)$ with the complex $e$ th roots of unity. If $\phi, \psi$ are the Brauer characters afforded by $U, W$ resp., then 


$$
\begin{aligned}
\chi_{H} & =\phi_{H}+\psi_{H}=\sum_{i=0}^{p-s-1} \alpha^{-(s / 2)-i}+\sum_{i=0}^{p-e+s-1} \alpha^{(s / 2)-i} \\
& =\alpha^{-s / 2}+\alpha^{s / 2}+(2 t-1) \rho
\end{aligned}
$$

where $\rho$ is the character of the regular representation of $H$.

Since $e$ is odd, there exists an automorphism $\sigma$ of the field of algebraic numbers which sends a primitive $e$ th root of unity to its square, and fixes the $p$ th roots of 1 . Exceptional $\chi$ must be fixed by $\sigma$, hence

$$
\alpha^{-s}+\alpha^{s}+(2 t-1) \rho=\left(\chi_{H}\right)^{\sigma}=\chi_{H}=\alpha^{-s / 2}+\alpha^{s / 2}+(2 t-1) \rho
$$

and so $\alpha^{-s}+\alpha^{s}=\alpha^{-s / 2}+\alpha^{s / 2}$. Then $s \equiv-s / 2(\bmod e)$ implies $3 s \equiv 0$ $(\bmod e)$, whence $3 \mid e$ and $e / 3 \mid s$. So $s$ even means $s=2 e / 3$. By [3, Theorem 1] with $s=u$, we have $4 e / 3=2 s \leqslant e+5$, whence $e \leqslant 15$. Therefore, $e=3,9$, or 15 .

(3.12) The case $e=15$ is impossible.

Proof. By [3, (3.9)] with $s=u=10$, we have

$$
2=9-[15 / 2] \leqslant t /(t-2),
$$

whence $t=4$. Now, [4, Theorem 2] with $x=5, s=10$ implies

$$
5=x \leqslant e-2 s+2 t=15-20+8=3 \text {, }
$$

a contradiction. (The methods of proof of Theorem 1 will also eliminate this case, without appealing to [4].)

(3.13) The case $e=3$ is impossible.

Proof. If $e=3$, then by (3.11), $U_{N}=V_{p-2}\left(\alpha^{-1}\right)$. Now [1, Lemma 3.3] implies $L_{1}\left(\leftrightarrow V_{3}(\alpha)\right)$ is a skew summand of $U \otimes U$, hence

$$
\operatorname{dim} L_{1} \leqslant(p-2)(p-3) / 2=((p-5) / 2) p+3 \text {. }
$$

But [3, Lemmas 2.1 and 2.2] show that $L_{1}$ lifts to an $R^{\prime}$-free, $R^{\prime} G$-module whose character contains exceptional characters in $B_{1}$ with total multiplicity at least $t-1$. Hence

$$
(2 t-3) p+4=(t-1)(2 p-3) \leqslant \operatorname{dim} L_{1} \leqslant((p-5) / 2) p+3,
$$

so that $2 t-3 \leqslant(p-7) / 2$ and $(p-1) / 3=t \leqslant(p-1) / 4$, a contradiction.

(3.14) The case $e=9$ cannot occur.

Proof. If $e=9$ then $U_{N}=V_{p-6}\left(\alpha^{6}\right), W_{N}=V_{p-3}\left(\alpha^{3}\right)$. In the symmetric summand of $U \otimes U, \alpha^{j}$ is a pmv $t / 2$ times if $j \equiv \pm 3$ or $\pm 1(\bmod e)$, $t / 2-1$ times otherwise. Furthermore (if $L_{i} \leftrightarrow V_{2 i+1}\left(\alpha^{i}\right)$ ), $L_{0}=1_{0}, L_{2}$ and $L_{4}$ are symmetric summands of $U \otimes U[1$, Lemma 3.3]. 
As in the proof of Theorem 1, each of $U$ and $W$ is a constituent of $L_{4}$ at least $t-1$ times [3, Lemmas 2.1, 2.2]. There exists an irreducible $K G$-module $M$ which absorbs $\alpha^{6}$ (the npmv of $U$ ). Furthermore, $U$ and $M$ are both incident to a common (nonexceptional) node in the graph of $B_{1}$, sep $M=$ rem $M \leqslant 5$, and the npmv of $M^{*}$ is $\alpha^{2}$ (see Proposition 2.6 and the proof of Proposition 2.3 in [3]). Thus $M \leftrightarrow V_{m}\left(\alpha^{m-3}\right), m=\operatorname{rem} M<5$ [1, Lemma 2.3].

Since $\alpha^{6}$ is a main value (and only a pmv) of the symmetric part of $U \otimes U$ exactly $t / 2$ times, [3, Lemma 2.7] implies $M$ is a constituent of $L_{4}$ with multiplicity at least $t / 2-1$. Now $\operatorname{dim} M>p$ [1, Theorem 5.7], so $M$ has a pmv $\gamma$. Also, $\alpha^{6}$ and $\gamma$ together occur as pmv's of $L_{4}$ at least $t-1$ times [3, Lemma 2.7], so $\gamma \neq \alpha^{6}$.

Since $V_{m}\left(\alpha^{m-3}\right) \otimes V_{5}\left(\alpha^{2}\right)$, and hence $M \otimes L_{2}$, has 1 as a npmv [1, Lemma 2.4], it follows by [1, Theorem 4.1] that

$$
\operatorname{Hom}_{K G}\left(M^{*}, L_{2}\right) \neq 0 \text {. }
$$

Therefore, $M$ (and $M^{*}$, if $M \approx M^{*}$ ) is a constituent of $L_{2} \approx L_{2}^{*}$. So $t / 2 \leqslant$ multiplicity of $M$ in $L_{2} \oplus L_{4} \leqslant$ multiplicity of $\gamma$ as a pmv of $L_{2} \oplus L_{4} \leqslant t / 2$. Hence, $M$ (and $M^{*}$, if $M \not M^{*}$ ) is a constituent of $L_{2} \oplus L_{4}$ with multiplicity exactly $t / 2$. Then $2 V_{p}(\gamma) \nmid M$, and $2 V_{p}(\gamma) \nmid M \oplus M^{*}$ if $M^{*} \approx M$. Also, $\alpha^{6}$ is a pmv of $L_{4}$ exactly $t / 2$ times, due to the presence of $U$ as a constituent, and $U$ must occur exactly $t-1$ times in $L_{4}$.

Since $V_{p}(\gamma)^{*}=V_{p}\left(\gamma^{-1}\right), \gamma^{-1}$ occurs as a pmv of $L_{2}$ whenever $\gamma$ does. So if $\gamma=\alpha^{3}$, then $\gamma^{-1}=\alpha^{6}$ is a pmv of $L_{2} \oplus L_{4}$ at least $t / 2+1$ times, a contradiction. Therefore, $\gamma=\alpha^{ \pm 1}$.

Suppose $M \approx M^{*}$. We have the configuration

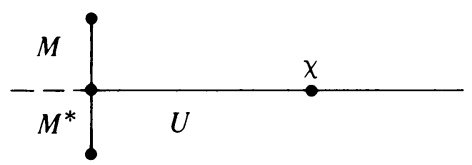

in the Brauer graph, so $m \leqslant 2 . M_{N}^{*}=V_{m}\left(\alpha^{2}\right) \oplus V_{p}\left(\alpha^{ \pm 1}\right)$. Since $G=G^{\prime},[1$, Lemma 2.3] forces

$$
1=\left(\alpha^{2} \alpha\right)\left(\alpha^{ \pm p} \alpha^{-p(p-1) / 2}\right)=\alpha^{3 \pm 1}
$$

if $m=2$, or $1=\alpha^{2 \pm 1}$ if $m=1$, in either case a contradiction. Therefore, $M \approx M^{*}$. Then $\alpha^{m-3}=\alpha^{2}$ implies $m=5$, whence $M \approx L_{2}$ and $M_{N}=$ $V_{5}\left(\alpha^{2}\right) \oplus V_{p}(\alpha) \oplus V_{p}\left(\alpha^{-1}\right)$. The Brauer graph now contains

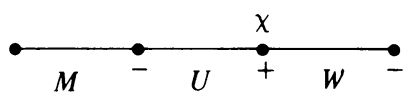


There is also an irreducible $K G$-module $X$ which absorbs $\alpha^{3}$ (the npmv of $W) . X$ and $W$ are both incident to a common (nonexceptional) node in the Brauer graph, sep $X=\operatorname{rem} X \leqslant 2$, and the npmv of $X^{*}$ is $\alpha^{5} . X$ has a pmv, say $\pi$. Since $\alpha^{6}$ is a pmv of $L_{4} \approx L_{4}^{*}$ exactly $t / 2$ times, so is $\alpha^{3}$. Then $X$ is a constituent of $L_{4}$ at least $t / 2-1$ times. Hence, $\pi \neq \alpha^{ \pm 3}, \alpha^{ \pm 1}$, as these pmv's have been produced as much as possible by $U, M$ and $W$. So $X$ has multiplicity exactly $t / 2-1$ as a constituent of $L_{4}$, hence [3, Lemma 2.7] implies $W$ has multiplicity exactly $t-1$.

$X \leftrightarrow V_{x}\left(\alpha^{x+3}\right), x \leqslant 2$. Suppose $X \not X^{*}$. We have the picture

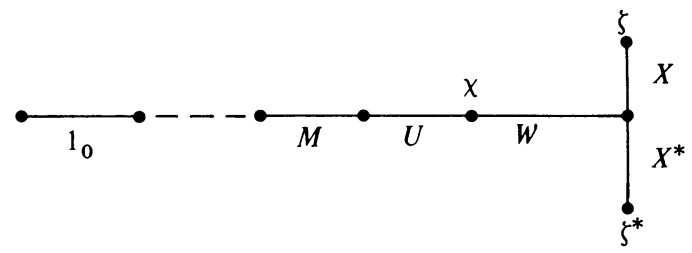

where sep $W=3$ implies $x=1$.

Let $X_{N}=V_{1}\left(\alpha^{4}\right) \oplus \sum_{i} V_{p}\left(\pi_{i}\right), \pi_{i} \neq \alpha^{ \pm 1}, \alpha^{ \pm 3}$. Then

$$
X_{N}^{*}=V_{1}\left(\alpha^{5}\right) \oplus \Sigma_{i} V_{p}\left(\pi_{i}^{-1}\right) \text {. }
$$

If we identify $\alpha(H)$ with the complex 9 th roots of unity, then $\zeta_{H}=n \rho_{H}+\alpha^{4}$ $+\sum \pi_{i}, n=t(\zeta(1)-1) / p$. Thus the irreducible constituents of $\zeta_{H}$ determine the main values of $X$.

Let $\sigma$ be an automorphism of the field of algebraic numbers which squares a primitive 9th root of 1 . Then $\zeta_{H}^{o}=n \rho_{H}+\alpha^{-1}+\Sigma \pi_{i}^{2}$. So if $\zeta^{\sigma}=\zeta$ or $\zeta^{*}$, then $\alpha^{-1}$ is a pmv of $X$ or $X^{*}$, a contradiction. Now $\left(\zeta^{\sigma^{2}}\right)_{H}=n \rho_{H}+\alpha^{7}+$ $\Sigma \pi_{i}^{4}$. If $\zeta^{\sigma^{2}}=\zeta^{*}$, then $\zeta^{\sigma^{4}}=\zeta$ implies $\zeta^{\sigma^{2}}=\zeta$ since $\sigma$ has order 6 . But if $\zeta^{\sigma^{2}}=\zeta$, then

$$
\zeta_{H}=n \rho_{H}+\alpha^{7}+\Sigma \pi_{i}^{4}=n \rho_{H}+\left(\alpha^{7}\right)^{4}+\Sigma \pi_{i}^{16} .
$$

Hence, $\alpha^{28}=\alpha$ is a pmv of $X$, a contradiction. It follows that $\zeta, \zeta^{\sigma}, \zeta^{\sigma^{2}}, \zeta^{*}$, $\left(\zeta^{*}\right)^{\sigma},\left(\zeta^{*}\right)^{\sigma^{2}}$ are six distinct characters in $B_{1}$, none of which lies on the real stem. The above configuration, with sep $M=5$, shows this is impossible.

We may assume that $X \approx X^{*}$. Then $X \leftrightarrow V_{2}\left(\alpha^{5}\right)$ and the graph now contains

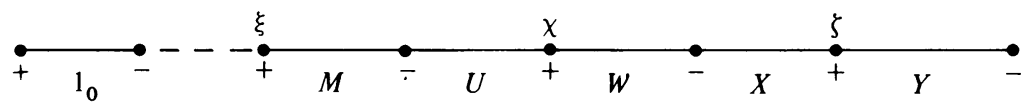

where $Y \leftrightarrow V_{p-1}\left(\alpha^{4}\right)$.

Suppose $\operatorname{dim} X=p+2$ and $\operatorname{dim} Y=p-1$. Then $V_{p}(\pi) \approx V_{p}(\pi)^{*}$ 
implies $\pi=1$. By [1, Lemma 2.2]

$$
\zeta_{H}=\alpha^{5}+\alpha^{4}+\alpha^{0}+2 t \rho_{H} .
$$

Thus, $\zeta$ has two algebraic conjugates in $B_{1}$, which must also lie on positive nodes on the real stem. But since $\zeta(1)=2 p+1, \chi(1)=2 p-9$ and $\xi(1) \geqslant$ $3 p+1$, there are not enough nodes available. Hence, we may assume $\zeta(1) \geqslant 3 p+1$.

Let $L_{4}^{\prime}$ be an $R^{\prime}$-free, $R^{\prime} G$-module with $L_{4}^{\prime} / J\left(R^{\prime}\right) L_{4}^{\prime}=L_{4}$. Since the multiplicity of $U$ and $W$ in $L_{4}$ is exactly $t-1$, [3, Lemma 2.2] implies the exceptional characters must occur in the character afforded by $L_{4}^{\prime}$ with total multiplicity exactly $t-1$. Then in order that $M$ and $X$ occur in $L_{4} t / 2-1$ times, each of $\xi$ and $\zeta$ has multiplicity $t / 2-1$ in the character of $L_{4}^{\prime}$. If $A$ denotes the symmetric part of $U \otimes U$, we have

$$
\begin{aligned}
p(5 t-9)+t+8 & =(t / 2-1)(6 p+2)+(t-1)(2 p-9) \\
& \leqslant(t / 2-1)(\xi(1)+\zeta(1))+(t-1) \chi(1) \\
& \leqslant \operatorname{dim} L_{4} \leqslant \operatorname{dim} A-\operatorname{dim} L_{2}-1 \\
& =(p-6)(p-5) / 2-(2 p+5)-1 \\
& =p(p-15) / 2+9,
\end{aligned}
$$

so that $5 t-9<(p-15) / 2$. Then $(p-1) / 9=t<(p+3) / 10$ and $p<$ 37. But $e=9$ and $t \geqslant 4$ imply $p \geqslant 37$, a final contradiction.

\section{REFERENCES}

1. H. I. Blau, Under the degree of some finite linear groups, Trans. Amer. Math. Soc. 155 (1971), 95-113.

2. , An inequality for complex linear groups of small degree, Proc. Amer. Math. Soc. 28 (1971), 405-408.

3. Finite groups where two small degrees are not too small, J. Algebra 28 (1974), 541-555.

4. Under the degree of some finite linear groups. II, Trans. Amer. Math. Soc. 203 (1975), 87-96.

5. R. Brauer, On groups whose order contains a prime number to the first power. I, Amer. J. Math. 64 (1942), 401-420.

6. W. Feit, Groups with a cyclic Sylow subgroup, Nagoya Math. J. 27 (1966), 571-584.

7. On finite linear groups, J. Algebra 5 (1967), 378-400.

8. _ Representations of finite groups, Part I, Lecture Notes, Yale University, New Haven, Conn., 1969.

9. B. Rothschild, Degrees of irreducible modular characters of blocks with cyclic defect groups, Bull. Amer. Math. Soc. 73 (1967), 102-104.

Department of Mathematical Sciences, Northern Illinois University, DeKalb, Illinois 60115 